\title{
The Thick and the Thin of It: Contextual Effects in Body Perception
}

\author{
Douglas H. Wedell and Elaine M. Santoyo \\ University of South Carolina \\ Jonathan C. Pettibone \\ Southern Illinois University Edwardsville
}

\begin{abstract}
This research investigated how and when exposure to different body contexts alters body ideals used in judgment. University students judged the width and pleasantness of human figures, with context manipulated by presenting mostly narrow or mostly wide forms. In Experiment 1, silhouettes were presented simultaneously on the page. In Experiment 2, figures were presented successively on a computer screen as detailed pictures. In both experiments, width ratings were consistent with A. Parducci's (1995) range-frequency theory, which predicted the same figure is judged thinner in the wide context. Pleasantness ratings were well described by an ideal-point model that used a gaussian similarity function, with the ideal shifting to a narrower width in the narrow context. The assimilative shifts in ideals with mere exposure to a small set of contextual stimuli supports the hypothesis that mass media presentations of ultrathin individuals may lead women to adopt a "thin ideal" in judging themselves and others. Although exposure to narrow or wide sets of figures had no consistent effect on measures of body satisfaction, individual differences in body satisfaction were related to ideal-point shifts. Women who were dissatisfied with their own bodies consistently used thin ideals to judge body images and were insensitive to the contextual manipulation of body image.
\end{abstract}

We live in a modern, mass-media culture that bombards us with images across various media formats: print, television, movies, and the Internet. Researchers examining the origins of eating disorders have been particularly struck by the media emphasis on excessive thinness for women and how exposure to these images may negatively affect women's self-perceptions (Kilbourne, 1994). Correlational analyses have established links between media exposure to the ultrathin images of women and eating disorders (Murray, Touyz, \& Beumont, 1996; Stice, Schupak-Neuberg, Shaw, \& Stein, 1994). Furthermore, experimental laboratory studies have demonstrated links between exposure to skewed body images and negative self-assessments in women (Kalodner, 1997; Stice \& Shaw, 1994). Some researchers have argued that the key mechanism linking exposure to thin body images and negative self-appraisals is that such exposure leads to the establishment of a "thin ideal" used in evaluating oneself (Stice, 1994). Although this mechanism is plausible, we contend that it has not been ex-

Requests for reprints should be sent to Douglas H. Wedell, Department of Psychology, University of South Carolina, SC 29208.

E-mail: Wedell@sc.edu amined directly. To do so requires demonstrating how exposure to thin body images leads to shifts in ideals using rigorous procedures for inferring ideals from judgments. The research we report provided a rigorous test of this mechanism. Like the previous research, we also examined individual self-assessments of own body satisfaction and explored the linkage of these measures to judgments.

\section{JUDGMENTAL RELATIVITY}

Our research is founded on the concept of judgmental relativity, reflected in the fact that the same figure can be judged wide in one context and narrow in another. This phenomenon is well described by Parducci's (1965, 1995) range-frequency theory of judgment, which assumes that the stimulus evokes comparisons to a distribution of automatically recruited contextual stimuli. This distribution is influenced by relevant stimuli that have occurred frequently, recently, or define endpoints on the continuum (Kahneman \& Miller, 1986). Accordingly, a stimulus will be rated high if its value falls near the top of the range, defined by the maximum stim- 
ulus in mind at the time of judgment (the range principle). Similarly, it will be rated high if its value ranks high in the contextual set (the frequency principle).

Range-frequency theory has successfully predicted the pattern of judgments in both psychophysical domains, such as judgments of numerosity or size (Birnbaum, 1974; Parducci \& Wedell, 1986; Wedell, 1996), and social domains, such as judgments of attractiveness, happiness, likableness, and performance (Mellers \& Birnbaum, 1983; Smith, Diener, \& Wedell, 1989; Wedell, 1994; Wedell, Parducci, \& Geiselman, 1987). However, contextual manipulations can also produce disordinal effects not explained by range-frequency theory. For example, Wedell and Pettibone (1999) showed that although contextual effects on judgments of the widths of facial features were well modeled by range-frequency theory, the effects of context on pleasantness judgments of the faces were not. Instead, those effects were strongly disordinal and consistent with a model in which the ideal used to evaluate pleasantness shifted systematically toward the average of contextual values.

Wedell and Pettibone (1999) demonstrated that context effects on ideals can be well described by a gaussian ideal-point model (i.e., a model in which the single-peaked functions describing participants' attitudes toward different stimulus values follow a normal curve function, with the function peaking at the ideal point). Results from that research could not distinguish between two mechanisms that might produce these shifts. One of these, the judgment-mediated model, assumes that the ideal is tied to a particular judgment value so that range-frequency effects on judgments mediate shifts in ideals. A second, the prototype-based model, assumes that biased retrieval of values from the recent context recruits a contextually influenced prototype that serves as an ideal. Within this framework, demonstration of independent context effects on attribute judgments and ideal-point locations would provide evidence for the prototype model over the judgment-mediated model.

\section{APPLICATION TO BODY IMAGES}

The concept that ideals guide evaluation has played a prominent role in the literature on body image, which assumes that people compare features of their own bodies to an ideal body image (Stice, 1994). Accordingly, people will negatively evaluate themselves to the degree that their own body features deviate from an ideal. Several researchers have speculated how mass media portrayals of body images may lead to an unreasonably thin ideal and corresponding negative self evaluation, especially for women (Henderson-King \& Henderson-King, 1997; Stice, et al., 1994). In support of this idea, researchers have noted how mass media portrayals of body images are very positively skewed, representing a preponderance of underweight individuals viewed in both print and television media (Fouts \& Burggraf, 1999; Spitzer,
Henderson, \& Zivian, 1999). Others have demonstrated how individuals exposed to this skewed distribution of body images may internalize a thin ideal and judge themselves more negatively (Botta, 1999; Thornton \& Maurice, 1997, 1999). Still others have demonstrated how the internalized thin ideal may contribute to eating disorders, such as bulimia and anorexia nervosa (McCarthy, 1990; Stice, 1994; Stice \& Shaw, 1994). These results support the idea that exposure to the ultrathin body images in the mass media lead women to dissatisfaction with their own bodies.

Our research examined more carefully the perceptual basis of this supposition. Research on the thin ideal cited earlier typically has used questionnaire items designed to measure satisfaction with one's body image as the dependent variable. Decreased satisfaction was then assumed to be due to a shift toward a thinner and less attainable ideal. This presumed shift in ideal has not been demonstrated directly. The studies reported herein used techniques for inferring body ideals from judgments of body images to determine the degree to which ideals shift with exposure to skewed sets of body images. Thus, we tested the assumption so often found in the literature that exposure to thin body images leads to a thin ideal that is used for evaluation.

Different researchers have argued for different bases for the shifting of ideals. For example, some have argued that positive reinforcers associated with thinner body images lead to this shift (Fouts \& Burggraf, 1999; Stice, 1998). Others have argued that the contrast between the glamorously portrayed thinner individuals and oneself leads to lower self-esteem (Thornton \& Maurice, 1999), a contrast effect rather than a shift in ideal. This research tested whether shifts in ideals can occur through mere exposure, parallel to the contextual effects demonstrated for judgments of facial configurations by Wedell and Pettibone (1999). To demonstrate this effect, we used simplified stimuli that did not confound, for example, thinness of the figures with the elegance or glamour of clothes, settings, or other related features. Shifts in ideals with mere exposure to a set of such simplified body images would provide evidence for a basic perceptual mechanism guiding the development and maintenance of a body ideal. Such a demonstration would not nullify alternative explanations based on reinforcement, but it would show that reinforcement is not necessary for such effects.

\section{EXPERIMENT 1: JUDGMENTS OF SILHOUETTES}

In Experiment 1, we presented participants with booklets containing pages of human silhouettes whose widths were distorted through reduction or enlargement along the horizontal axis. The effects of presenting ultrathin or ultrawide versions of the silhouettes were then assessed on descriptive judgments of width and evaluative judgments of pleasantness of six target silhouettes common to all contextual distributions. This ma- 
nipulation allowed us to examine how standards for judging body images change with context. Of course, the increase in experimental control associated with using silhouettes comes at a cost of generalizing to more typically viewed body images. Thus, in Experiment 2 we attempted to generalize our results to more realistic-looking body images.

The use of simultaneous presentation of several body images on a page was designed to enhance the effects of context on judgment, as demonstrated in psychophysical and social research (Parducci \& Wedell, 1986; Wedell \& Parducci, 1988). Once again, this manipulation may afford greater experimental control over the context at the cost of generalizing to the more typical situation in which body images are viewed one at a time. In Experiment 2 we addressed this issue by using successive rather than simultaneous presentation of images.

Finally, because past research has focused on effects of exposure on individual's self-perceptions, we included a questionnaire at the end of the booklets designed to evaluate self-perceptions and physical attributes that might moderate judgments. If previous research generalizes to our current experimental paradigm, then we would expect a tendency for individuals exposed to the positively skewed distribution of body images to rate their satisfaction with their own body image lower. Research on how characteristics of the self affect judgments of others also suggests that the questionnaire data may provide insights into individual differences in the body judgment tasks (Lambert \& Wedell, 1991).

\section{Method}

Participants and design. Participants were 120 undergraduates (41 men, 78 women, and 1 whose gender was unspecified), drawn from the University of South Carolina psychology department participant pool, who volunteered in exchange for course credit. The basic design consisted of the factorial combination of four between-subjects variables. Two of these variables-range (restricted or full) and skew (positive or negative) - combined to define the four distributions of images that were rated. The range variable determined whether participants saw a full or restricted range of body images, and the skew variable determined whether they saw a preponderance of thin or thick body images. The other two between-subjects variables were concerned with order effects. Judgment order determined whether participants made evaluative judgments of pleasantness or descriptive judgments of width first. Body type order represented the three different counterbalanced orders in which participants could view the three body types included in the study.

Three additional variables were factorially manipulated within subjects: (a) body type, (b) target stimuli, and (c) type of judgment. Body type corresponded to three different silhouette profiles. Target stimuli were six width levels that were common to all distributions. Type of judgment corresponded to ratings of widths and pleasantness.

Materials. To examine the generality of results across different types of bodies, we used three body types, generated by converting photographs of women into silhouettes. The three body types correspond to a thin $(T)$, a medium $(M)$, and a wide $(W)$ figure. The original silhouette was designated size 13 and represented $100 \%$ of original size. We created additional sizes by increasing or decreasing the width by multiples of 3\%, so that the final set of 25 sizes ranged from $64 \%$ to $136 \%$ of the original width. The distribution of body widths shown to participants in each of the four contextual conditions are presented in Table 1. Sizes 5, 9, 11, 13, 15, and 20 were the six target sizes common to all distributions. The four distributions were designated restricted range-positive skew $(R P)$, full range-positive skew, restricted range-negative skew $(R N)$, and full range-negative skew.

Each distribution of 12 body images was printed in black on a single sheet of $8 \frac{1}{2} \times 11$ in. $(22 \mathrm{~cm} \times 28 \mathrm{~cm})$ paper, with corresponding instructions printed on the top of the page. An example of one of the pages for the medium target in the $R P$ condition is shown in Figure 1, with scale values placed in the boxes where participants rated the silhouettes. There were three rows of four columns each, which were blocked off in $4.3 \times 6.8 \mathrm{~cm}$ rectangular frames approximately $0.5 \mathrm{~cm}$ apart and in which the body images, approximately $6.6 \mathrm{~cm}$ tall, were printed. A $0.8-\mathrm{cm}^{2}$ box was placed in the lower left-hand corner of each frame and served as a place for participants to record their ratings. A $0.5-\mathrm{cm}$ vertical line at the top of each frame was used to center the body image. Referring to locations on the page from left to right and top to bottom, the target stimuli always appeared at locations 2,4 , $5,7,10$, and 12 , with contextual stimuli in the other locations. Six different random assignments of the target stimuli to these locations were created for each body type along with

TABLE 1

Stimulus Values for the Four Distributions Used in Experiments 1 and 2

\begin{tabular}{|c|c|c|c|c|c|c|c|c|c|c|c|c|c|c|c|c|c|c|}
\hline Distribution & \multicolumn{18}{|c|}{ Sizes } \\
\hline \multicolumn{19}{|l|}{ Restricted range } \\
\hline Positive skew & 1 & 2 & 3 & 4 & 5 & 6 & 7 & 9 & 11 & 13 & 15 & & & 20 & & & & \\
\hline Negative Skew & & & & & 5 & & & 9 & 11 & 13 & 15 & 18 & 19 & 20 & 22 & 23 & 24 & 25 \\
\hline \multicolumn{19}{|l|}{ Full range } \\
\hline Positive skew & 1 & 2 & 3 & 4 & 5 & 6 & & 9 & 11 & 13 & 15 & & & 20 & & & & 25 \\
\hline Negative skew & 1 & & & & 5 & & & 9 & 11 & 13 & 15 & & 19 & 20 & 22 & 23 & 24 & 25 \\
\hline
\end{tabular}



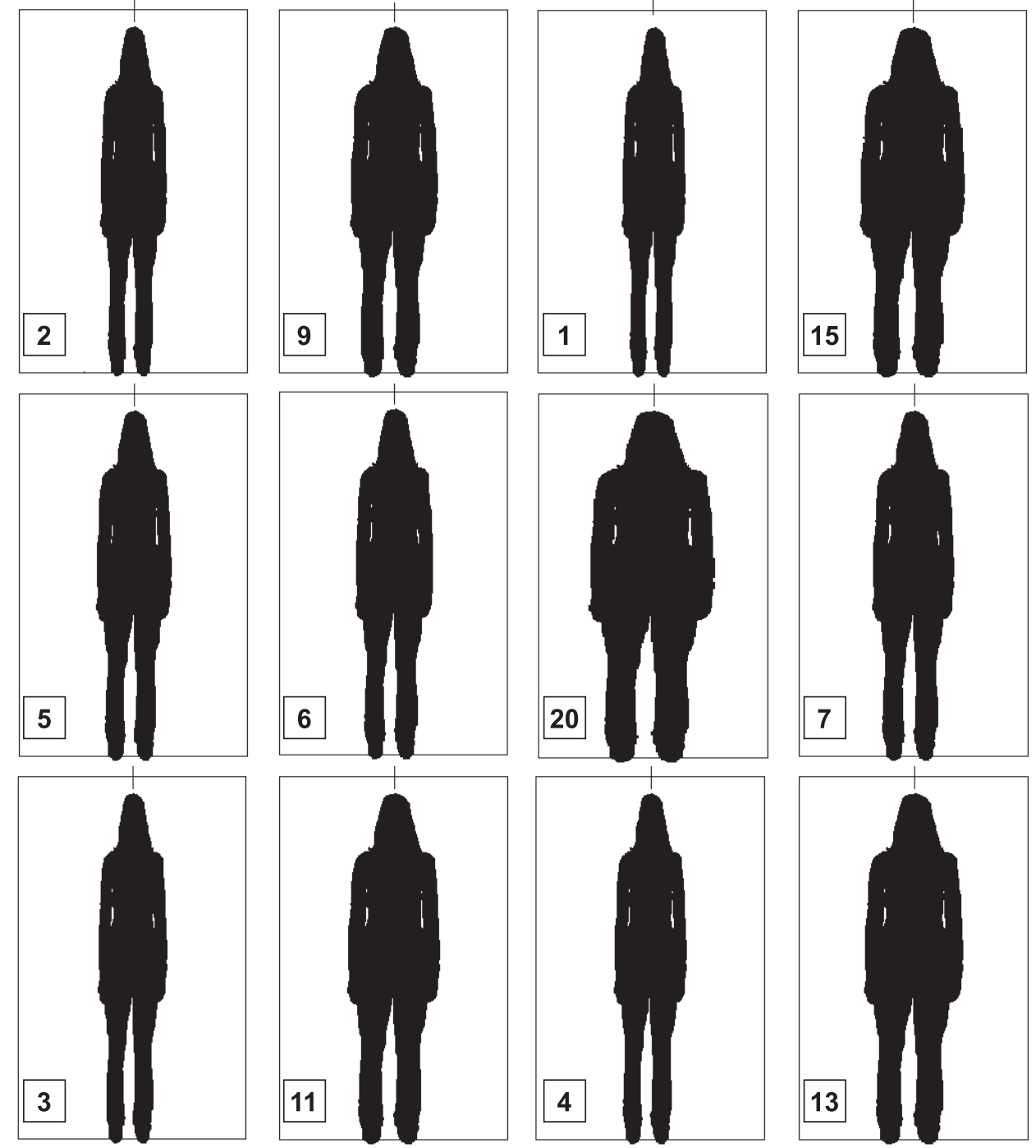

FIGURE 1 The 12 body images presented in the restricted range-positively skewed condition for the medium body type. The arrangement is representative of how the images appeared to participants for rating in Experiment 1. Numbers indicate corresponding sizes for the silhouettes and appear in the boxes where participants would have marked their ratings.

corresponding random assignments of the contextual stimuli to locations.

Each booklet consisted of 8 pages. A dark cover page prevented viewing of subsequent pages. Six pages of different sets of body images followed. Stimuli for a given booklet were always drawn from the same distribution (RP, RN, full range-positive skew, or full range-negative skew). The assignment of body types to pages was counterbalanced, and each page used a different random arrangement of targets on the page. For half the booklets, instructions for the first 3 pages of silhouettes asked participants to rate width, and instructions for the last 3 pages of silhouettes asked participants to rate pleasantness. The remaining booklets reversed the order of the judgment tasks.

Instructions for rating body images appeared at the top of each page along with a 9-point scale. For the descriptive rat- ing task, participants were told to rate the widths of the figures, with 1 designated very thin in appearance and 9 designated very thick in appearance. For the pleasantness rating task, participants were asked to judge how pleasant the human silhouette appeared to them on a similar 9-point scale, with 1 designated very unpleasant in appearance and 9 designated very pleasant in appearance.

The final page of the booklet consisted of a questionnaire containing 19 questions about the participant's actual weight; ideal weight; height; gender; and attitudes regarding his or her body, weight, and activities related to health. Questions examining global satisfaction with body image and weight were similar to those used in body image research (Brown, Cash, \& Mikulka, 1990; Cash, 1994; Stice \& Shaw, 1994). Attitude items were evaluated on a 5-point agree-disagree scale with 1 designated strongly agree and 5 designated strongly disagree. 
Procedure. Participants were tested in groups of up to 25 in a large classroom. After filling out informed consent forms, participants were instructed that they would be judging the silhouettes on two different rating scales, one concerned with perceptions of widths of the figures and the other concerned with the pleasantness of the figures. They were told to pay careful attention to the instructions at the top of each page to be certain they were using the correct rating scale.

\section{Results}

Participants who failed to show a positive relationship between figure width and width ratings for target stimuli were assumed to have misunderstood the instructions (most likely judging pleasantness rather than width). On the basis of this criterion, data from 9 of the 120 participants were excluded from analyses of the judgment data.

Analysis of width ratings. The width ratings for the three body types under the four different contextual conditions are presented in Figure 2. The higher ratings for the target stimuli in the positively skewed distribution (unfilled circles) than in the negatively skewed distribution (filled circles) is evident throughout and is consistent with the basic predictions of range-frequency theory. We conducted an analysis of variance (ANOVA) on the mean of the ratings of the six target stimuli across the three body types, with skew (positive or negative), range (full or restricted), and judgment order (width first or pleasantness first) as between-subjects
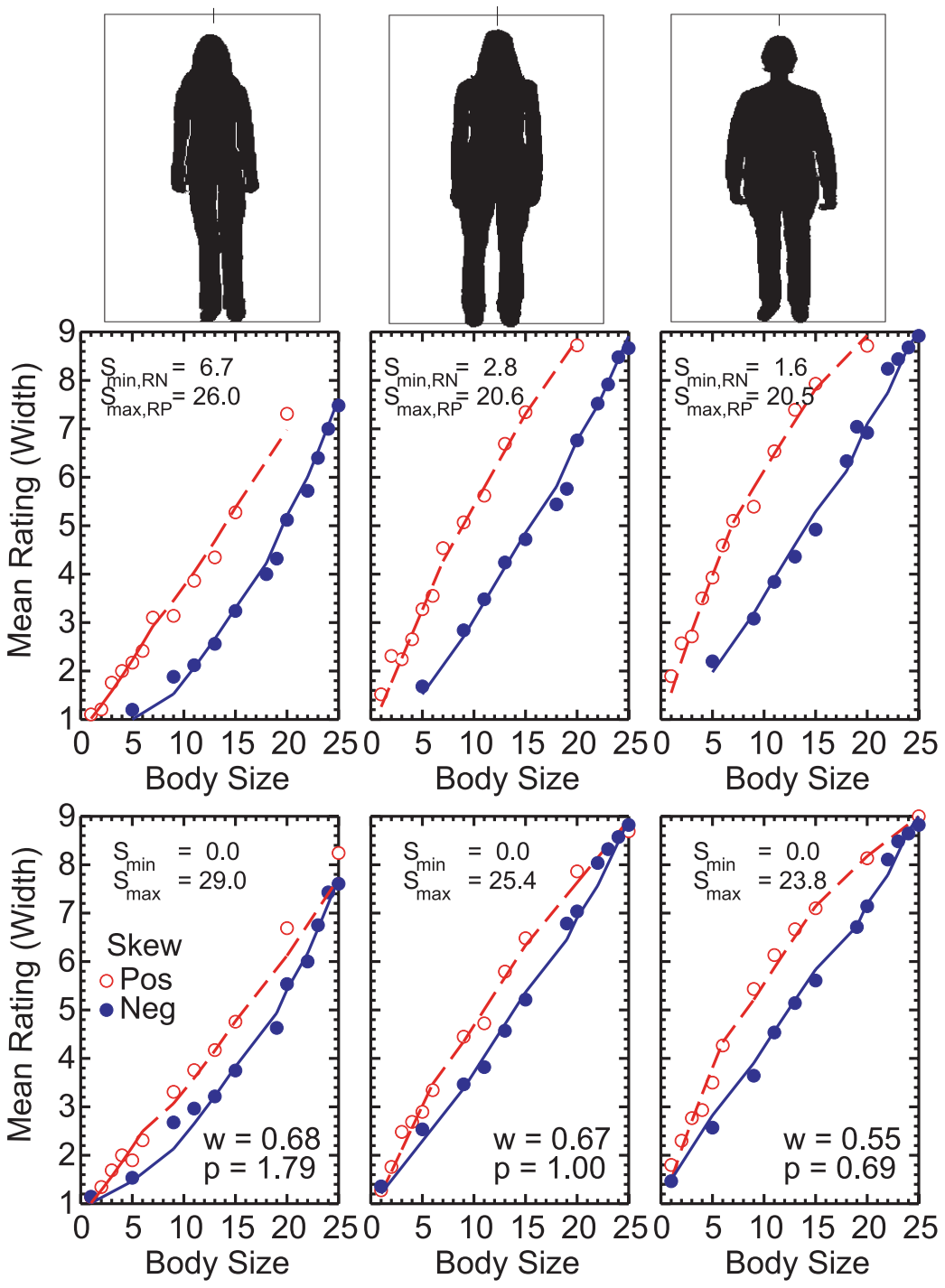

FIGURE 2 Mean ratings of the width of the body images presented in positively skewed (unfilled circles) and negatively skewed (filled circles) distributions. The lines represent the fit of the range-frequency model. Top rows correspond to the restricted-range condition, bottom rows correspond to the full-range condition, and columns correspond to different body types (from left to right, these are thin, medium, and wide types). The large effects of skewing shown in each panel correspond to the shifting judgment standards predicted by range-frequency theory. $\min =$ minimum; $\max =$ maximum; $R N$ $=$ restricted range-negative skew; $R P=$ restricted range-positive skew; $P o s=$ positive; Neg = negative; $w=$ range weighting value; $p=$ power exponent. 
factors. The main effect of skew was significant, $F(1,103)=$ $170.8, p<.001$, and reflected strong overall contrast effects: The same silhouette was rated thinner in the negatively skewed context than in the positively skewed context. The only other source to achieve significance was the Skew $\times$ Range interaction, $F(1,103)=18.7, p<.001$, reflecting stronger effects of skewing when the range was restricted.

To examine how well the range-frequency model fit the descriptive rating data, a version of the model with six free parameters was fit to the 48 mean ratings for each of the three body types. The following equation from Wedell (1996) was used to fit the data:

$$
C_{i k}=1-8\left[w \frac{\Phi_{i}^{p}-\Phi_{\mathrm{MIN}, k}^{p}}{\Phi_{\mathrm{MAX}, k}^{p}-\Phi_{\mathrm{MIN}, k}^{p}}+(1-w) \frac{\mathrm{rank}_{i k}-1}{N_{k}-1}\right]
$$

In Equation $1, C_{i k}$ represents the mean category rating of stimulus $i$ in context $k$, and the expression within the brackets represents the range-frequency judgment of the stimulus on an implicit $0-1$ scale. The additive constant, 1 , and multiplicative constant, 8 , simply serve to place the judgments on the appropriate 9-point rating scale. The physical width of stimulus $i$ is designated $\Phi i$, with $p$ representing the exponent of the psychophysical power function relating physical width to psychological impressions of width. The range value of the stimulus is represented by the first fraction, which subtracts the value of the minimum stimulus brought to mind at the time of judgment from the current stimulus value and divides by the range of values. The frequency value is represented by the second fraction, which subtracts the minimum rank of 1 from the rank of the current stimulus and divides by the range of ranks. The relative weighting range values over frequency values is given by $w$, with 0.5 representing equal weighting.

In fitting the contextual variations in ratings for a given body type, the range weighting value, $w$, and the power exponent, $p$, were held constant. Furthermore, the minimum and maximum values defining the range were held constant in the full-range conditions, and only two additional values were fit to the restricted-range conditions, for a total of 18 parameters fit to the 144 data points of Figure 2. The close adherence of the data points (circles) to the predicted values (lines) in Figure 2 demonstrates the excellent fit of the range-frequency model with the proportion of predicted variance varying between .988 and .992. The parameter values for the full-range conditions are shown in the bottom panels. The value of $w$ was nearly the same for the thin and medium figures but was somewhat smaller for the wide figure, indicating greater effects of skewing for the wide body type. The differences in power coefficients inferred for the three body types indicated different psychological scales for the three body types. The inferred minimum and maximum values defining the range were close to those actually presented in the full-range condi- tion (1 and 25) except for the thin body type, in which the maximum was substantially higher. As expected, the inferred values for the restricted end of the range shifted toward the actual values shown in that condition. Overall, the range-frequency model fit the data very well and provides a basis for a psychological interpretation of the differential effects of context.

Analysis of pleasantness ratings. The evaluative ratings of pleasantness for the three body types under the four different contextual conditions are presented in Figure 3. The rating functions exhibit the usual single-peaked form found in most attitude domains, with the preferred width (or ideal point) being at an intermediate value, and preference falling off as a function of distance from the ideal. Consistent with previously observed contextual effects on ideal-point preference (Riskey, Parducci, \& Beauchamp, 1979; Wedell \& Pettibone, 1999), the ideal point was located at a narrower value when the distribution was positively skewed, providing direct evidence for the hypothesis under investigation. For each participant, an ideal was computed by determining which target stimulus value corresponded to the highest pleasantness rating. If more than one target stimulus were assigned to the highest rating, these values were averaged together. The overall ideal was then the average of ideals computed for the three body types.

We conducted an ANOVA to determine whether ideals differed as a function of the three between-subjects variables: (a) skew, (b) range, and (c) judgment order. The main effect of skew was significant, $F(1,103)=12.0, p<.001$, reflecting the narrower ideal in the narrower context. The Skew $x$ Range interaction was marginally significant, $F(1,103)=$ $3.6, p<.07$, reflecting the reduced contextual influence for ideals in the full-range condition. The shifts in ideals shown in Figure 3 produce disordinal context effects, or preference reversals, in both the restricted- and full-range conditions. As an example, the pleasantness rating of target sizes 11 and 15 for the thin body type were submitted to a $2 \times 2$ repeated measures ANOVA separately for full and restricted ranges. In both cases, the significant Target $\times$ Skew interaction $(p<$ $.05)$ reflected a disordinal interaction in which preferences for the two body sizes were reversed. For restricted-range conditions, Target 11 was preferred to Target 15 in the positively skewed context $\left(M_{11}=6.7\right.$ vs. $\left.M_{15}=6.1\right)$, but the reverse was true in the negatively skewed context $\left(M_{11}=5.8\right.$ vs. $\left.M_{15}=7.0\right)$. Similarly, in the full-range condition Target 11 was preferred in the positively skewed context $\left(M_{11}=7.0 \mathrm{vs}\right.$. $\left.M_{15}=6.3\right)$ but not in the negatively skewed context $\left(M_{11}=6.5\right.$ vs. $M_{15}=7.5$ ).

Following Wedell and Pettibone (1999), we used a gaussian ideal-point model to fit the data. The equation used to fit the data was as follows:

$$
C_{i k}=1+b_{k} \exp \left[-c_{k}\left(\Phi^{p_{i}}-I_{k}\right)^{2}\right]
$$




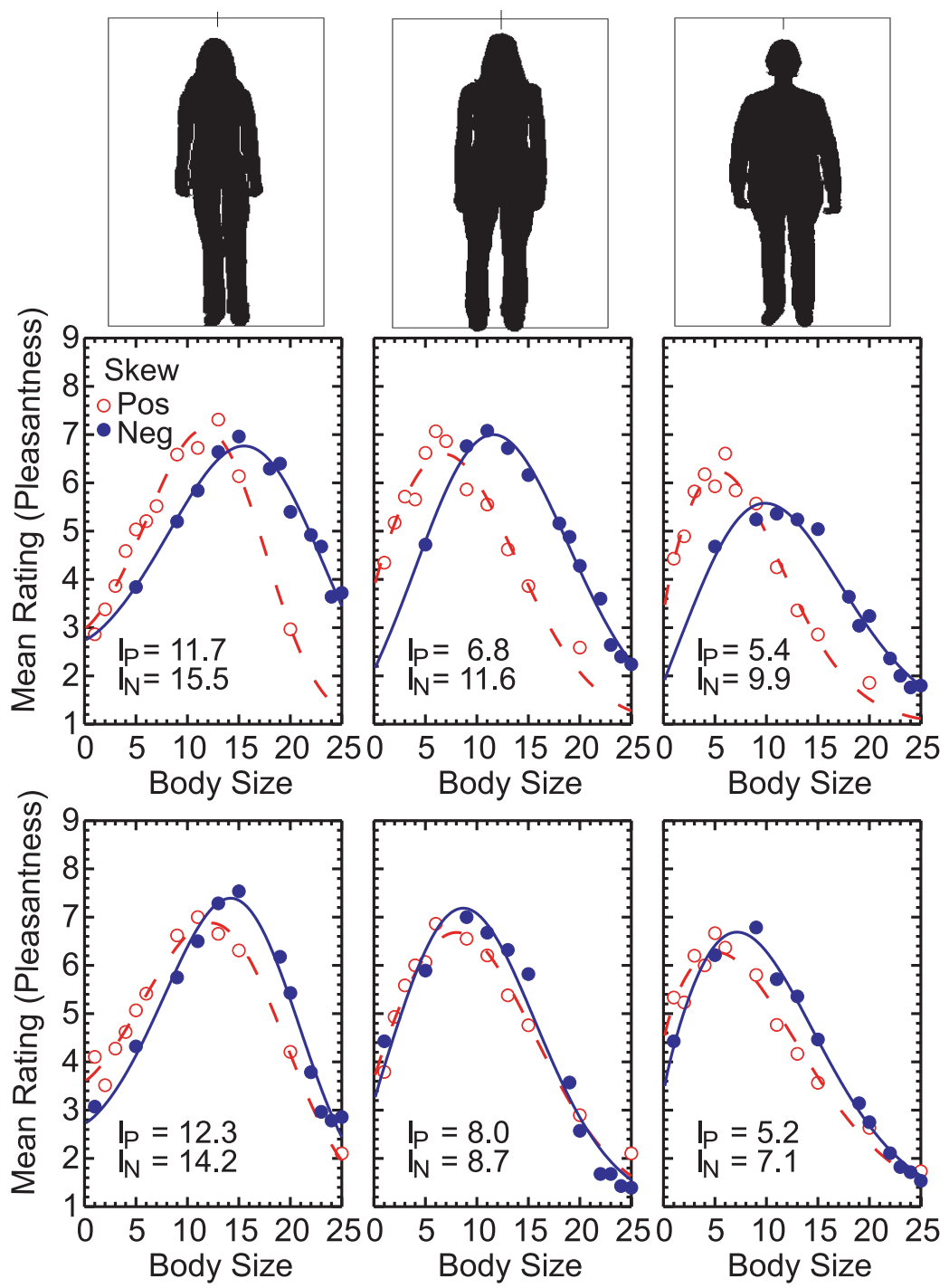

FIGURE 3 Mean ratings of the pleasantness of the body images presented in positively-skewed (unfilled circles) and negatively-skewed (filled circles) distributions. The lines represent the fit of the gaussian ideal-point model. Top rows correspond to the restricted-range condition, bottom rows correspond to the full-range condition, and columns correspond to different body types. The shifts in the peaks of the functions with skewing show how ideals for judging body images shift with context. $P o s=$ positive; $N e g=$ negative. Note $I_{P}$ and $I_{N}$ represent the inferred ideals for positive and negative skew, respectively.

with the mean category rating of stimulus $i$ in context $k$ being described by a linear function of the similarity of the stimulus to the ideal in that context. In Equation 2, the preference function follows a normal curve on the psychological scale defined by a power function, with power exponent $p$. The parameter $b_{k}$ defines the height of the preference function in context $k$, and parameter $c_{k}$ defines the width of the function in that context. Preference is assumed to fall off as a gaussian function of the distance between the stimulus and the ideal, $I_{k}$.

In fitting the data, we assumed a single value of $p$, the power exponent, for each body type and allowed $b, c$, and $I$ to vary with contextual condition. This meant that the 48 mean ratings for each body type were fit using 13 free parameters. Although a more parsimonious fit could be achieved by equating some of the parameter values across contextual conditions, we choose to fit the full model and use it as a descriptive tool. The fitted parameter values are presented in Table 2. The model provided a good account of the data, with the proportion of variance in mean ratings accounted for by the model varying between .973 and .983 .

The differences in inferred ideals across contexts were greater in the restricted-range conditions than in the full-range conditions for each of the three body types. Although the shift in ideals with skewing occurred for all three body types, there were variations in the magnitude of this effect. The parameter values in Table 2 can be used to index this and other effects operating on the preference curves. Note that because the scales change across body type, it is not possible to directly compare across body types the values of 
TABLE 2

Parameter Values for the Gaussian Ideal-Point Model Fit to the Four Contextual Conditions for Each Body Type, Experiment 1

\begin{tabular}{|c|c|c|c|c|c|c|c|c|c|c|c|c|}
\hline \multirow[b]{2}{*}{ Parameter } & \multicolumn{4}{|c|}{ Thin Body Type } & \multicolumn{4}{|c|}{ Medium Body Type } & \multicolumn{4}{|c|}{ Wide Body Type } \\
\hline & $R P$ & $R N$ & $F P$ & $F N$ & $R P$ & $R N$ & $F P$ & $F N$ & $R P$ & $R N$ & $F P$ & $F N$ \\
\hline$p$ & 1.282 & & & & 0.871 & & & & 0.750 & & & \\
\hline$c$ & 0.0021 & 0.0011 & 0.0013 & 0.0015 & 0.0242 & 0.0239 & 0.0205 & 0.0245 & 0.0685 & 0.0567 & 0.0388 & 0.0479 \\
\hline$b$ & 6.110 & 5.764 & 5.880 & 6.392 & 5.593 & 5.999 & 5.683 & 6.185 & 5.231 & 4.577 & 5.271 & 5.689 \\
\hline$I$ & 11.67 & 15.50 & 12.26 & 14.20 & 6.84 & 11.58 & 8.04 & 8.67 & 5.43 & 9.87 & 5.15 & 7.14 \\
\hline
\end{tabular}

Note. $\mathrm{RP}=$ restricted range-positive skew; $\mathrm{RN}=$ restricted range-negative skew; $\mathrm{FP}=$ full range-positive skew; $\mathrm{FN}=$ full range-negative skew, $p=$ power coefficient, $c=$ scaling coefficient inversely related to width of function, $b=$ multiplicative constant directly related to height of function, $I=$ ideal-point value.

TABLE 3

Principal Components Analysis (PCA) Loadings on First Component for Questionnaire Items Used in the Final Measure of Body Image

\begin{tabular}{ll}
\hline PCA Loading & \multicolumn{1}{c}{ Item } \\
\hline-.75 & I am satisfied with my weight. \\
-.66 & I think my weight is healthy. \\
.61 & Weight is an important concern in my life. \\
.67 & I frequently think about my weight. \\
.79 & I diet often. \\
.77 & I exercise primarily for the purpose of losing weight. \\
-.76 & I am satisfied with my body. \\
.61 & Others think I should decrease my weight. \\
\hline
\end{tabular}

the $c$ parameter, which is inversely related to the width of the preference function. The value of $b$, which relates to the height of the preference function, changed with body type, reflecting the descending preference from the thin to the medium to the wide body type.

Analyses related to the questionnaire measures. Loadings on the first principal component for the 19 questionnaire items with 117 participants who had no missing data were examined. A final measure of body image satisfaction was then based on summing 8 items with absolute loadings of .60 or higher (after first reversing items with negative loadings). The items, along with their loadings, are presented in Table 3 . We subtracted 24 from the total so that a score of 0 represented neutral endorsement of a positive body image, and scores could range from -16 (representing strong dissatisfaction with body image) to 16 (representing strong satisfaction with body image). Body image scores had a mean of 4.2 , a standard deviation of 6.7, and a reliability of .86, as estimated by coefficient alpha. Body image scores were significantly higher for men $(M=6.3)$ than for women $(M=3.0)$, $t(106)=2.5, p<.05$.

We conducted a correlational analysis separately for men and women to see how participant variables interrelated and how these related to the skewing manipulation. These correlations are presented in Table 4. The variables included the body image score derived from the questionnaire (BIMAGE), the participant's reported weight (WEIGHT),
TABLE 4

Correlations Among Key Participant and Judgment Variables for Women and Men, Experiment 1

\begin{tabular}{lccccc}
\hline \multicolumn{1}{c}{ Variable } & 1 & 2 & 3 & 4 & 5 \\
\hline Women $(N=69)$ & & & & & \\
1. BIMAGE & - & & & & \\
2. OVERWGHT & $-.58^{* * * *}$ & - & & & \\
3. WEIGHT & $-.50^{* * * *}$ & $.85^{* * * * *}$ & - & & \\
4. IDEALDEV & $.25^{* *}$ & .09 & .17 & - & \\
5. skew & $.24^{* *}$ & .14 & -.10 & .05 & - \\
Men $(N=38)$ & & & & & \\
1. BIMAGE & - & & & & \\
2. OVERWGHT & -.24 & - & & & \\
3. WEIGHT & $-.32^{* * *}$ & $.55^{* * * *}$ & - & & \\
4. IDEALDEV & -.10 & .04 & $.36^{* * * *}$ & - & \\
5. Skew & -.26 & .05 & .00 & -.08 & -
\end{tabular}

Note. $\quad$ BIMAGE $=$ body image scale OVERWGHT = perception of being overweight (actual weight minus ideal weight); WEIGHT = participant's actual weight; IDEALDEV = deviation score of ideal weight inferred from participant's judgments and average ideal in the corresponding contextual condition.

$* * p<.05 . * * * p<.01 . * * * * p<.001$.

the difference between actual and desired weight (OVERWGHT), the skewing condition, and a measure of how the ideal inferred for the participant compared with the mean ideal in that judgment condition (IDEALDEV). Low IDEALDEV scores indicate that the individual tended to prefer a thinner ideal than the others who were in that contextual condition.

In contrast with previous research (Stice \& Shaw, 1994; Thornton \& Maurice, 1999), female participants exhibited significantly higher body satisfaction after viewing the ultrathin silhouettes in the positively skewed distributions ( $M$ $=4.40$ ) than after viewing the negatively skewed distributions $(M=0.93)$, as indicated by the significant correlation between skew and BIMAGE. Men tended to exhibit the opposite effect, although this difference did not achieve statistical significance. No other measures correlated with skew.

The interrelations among the BIMAGE, WEIGHT, and OVERWGHT measures were as expected for both groups. Participants with higher body image scores reported weighing less and reported themselves as less overweight. This was 
true for both men and women, although the strength of these relations was greater for women. The significant positive correlation between BIMAGE and IDEALDEV measures for women is particularly interesting because it demonstrates how those who were dissatisfied with their own bodies used a thinner ideal in judging the silhouettes across the four conditions. The only other significant correlation was between WEIGHT and the IDEALDEV measure, in the positive direction, for men only. This correlation suggests that heavier men tended to prefer wider silhouettes.

\section{Generality of judgment results across groups.}

Given that the participant variables correlated with the ideals used to judge the experimental stimuli, we may ask to what extent the judgment results reported for the group as a whole reflect subgroups within our sample. In particular, we identified three subgroups: (a) women with lower body image scores (BIMAGE $<3.0, n=32$ ), (b) women with higher body image scores (BIMAGE 3.0, $n=38$ ), and (c) men $(n=39)$. We conducted ANOVAs on the mean width judgments of the target stimuli and on the inferred ideals for each group. In the domain of width judgment, each group showed a significant main effect of skewing and a significant Skewing $\times$ Range interaction, consistent with results reported earlier for the group as a whole. In the domain of pleasantness judgments, two of the groups showed significant effects of skewing on inferred ideals, consistent with the results reported earlier. However, the women with low body image scores showed no effects of skewing on inferred ideals $(F<1.0)$. This group appeared to use the same narrow standards to judge the pleasantness of body images regardless of the contextual set.

\section{Discussion}

The results from Experiment 1 provide clear evidence that even a brief exposure to a set of body images can quickly change the standards by which people judge those images. Descriptive judgments of the widths of the silhouettes showed the usual contrast effects found in so many other domains (Parducci, 1995): The same silhouette was rated thinner when it appeared among predominantly wider silhouettes. Context effects for width ratings were well described by range-frequency theory. In accordance with the theory, the effects of skewing the distribution of body images were greatest when the range was restricted. When the full range of body images was presented, context effects were significantly reduced, but not eliminated. This suggests that presentation of even a relatively few images at each extreme may stabilize judgments.

Consistent with research in psychophysical and social domains (Riskey et al., 1979; Wedell \& Pettibone, 1999), the contextual manipulations also led to shifts in ideal-point locations used to make evaluative ratings of pleasantness of the body images. Thus, when the context consisted of mostly thin silhouettes, a thinner ideal was adopted in judging these body images (as reflected in the rating function peaking at a thinner body image). It is important to note that this result provides experimental support for the oft-cited supposition that mass media exposure to ultrathin body images may result in the adoption of a thinner ideal for judging body images. As with the width ratings, the effects of skewing the distribution on pleasantness ratings were greater when the range was also restricted. The effects of context on pleasantness ratings were well described by a gaussian ideal-point model in which the ideal by which attractiveness is gauged shifts toward the mean of the contextual distribution of body images. These effects were particularly dramatic because they produced crossover interactions on the pleasantness function and therefore reversals of preference. Thus, an individual who finds her own body image more attractive than a thinner version of herself may, after exposure to a set of very thin body images, prefer the thinner version and become dissatisfied with her current body image. Because presenting the full range greatly reduced the shift in ideals, we speculate that similar representation of the full range of body images in the mass media may help reduce effects of skewing.

It is somewhat surprising that exposure to the wider sets of silhouettes rather than the thinner sets resulted in lower body satisfaction measures for the women in this study. This result is inconsistent with that reported by Stice and Shaw (1994). In their study, female undergraduates were exposed to magazine pictures with ultrathin models, average-sized models, or no models at all. The authors found that exposure to the ultrathin models produced, among other reactions, greater dissatisfaction with their own body image. The opposite finding in this study may be due to any of a number of procedural differences between the two studies. One of these differences is that we used silhouettes rather than actual photographs. We did this to both facilitate the manipulation of body width as well as avoid confounding width of image with other features of the image, for example, its perceived glamour. It is possible that the original finding by Stice and Shaw (1994) was due not to female participants adopting thinner ideals by which to judge themselves more negatively but to greater feelings of inadequacy when comparing themselves to the thin models on other dimensions; this is consistent with the contrast explanation provided by Thornton and Maurice (1997, 1999). Furthermore, it should be noted that Henderson-King and Henderson-King (1997) found individual differences in the effects of exposure to different body images on women's self-perceptions, with thinner women actually showing positive effects of exposure to thin body images and heavier women showing negative effects. Despite these questionnaire results, the rating data provide clear support for Stice and Shaw's assertion that exposure to thin body images facilitates the adoption of a thin ideal, which may then in turn lead to lower levels of satisfaction with one's own body image.

Finally, the analyses of judgment data segregated by group suggest that women with poor body images consis- 
tently used thin ideals to judge the silhouettes and were not sensitive to the contextual manipulation. Because they showed the usual context effects on judgments of widths of these same silhouettes, the insensitivity of their ideals to the contextual manipulation was not due to generalized contextual insensitivity; instead, they appear to have developed entrenched negative attitudes toward their own body image that may be sustained by the consistent use of ultrathin ideals. The dissociation of context effects on width judgments and pleasantness judgments for this group supports the prototype model of contextual shifts in ideals. If the judgment-mediated model were correct, then one would expect that the large contextual effects on width judgments demonstrated for this group would translate into similar shifts in ideal-point locations. The lack of contextual shifts in ideal-point locations for this group may then reflect greater stability of prototypical body images for women in this group, perhaps through repeated or compulsive retrieval of ultrathin images. Thus, satisfaction with one's own body image appears to be an important moderator of the effects of context on ideals.

\section{EXPERIMENT 2: JUDGMENTS OF MORE REALISTIC BODY IMAGES}

Although the use of silhouettes in Experiment 1 may have enhanced experimental control, it also limited the generality of the findings. It is unclear whether the same type of contextual processes would apply to judgments of more realistic figures. In Experiment 2 we tested the generality of Experiment 1 results by using more realistic, computer-generated figures. The six target images used in Experiment 2, which were generated using the three-dimensional computer software program Poser (version 4.0), are presented in Figure 4. Each of the figures differed only in width, with the same female figure attired in the same way in each case. Thus, we retained the experimental control associated with varying only a single aspect (width), but we used much more realistic figures that would be more likely to invoke retrieval and comparison with memories of people participants have encountered throughout their lives.

Because we used the same basic stimulus design as in Experiment 1 , the results of the two experiments are directly comparable. However, Experiment 2 differed from Experiment 1 in ways other than simply the use of the more realistic-looking figures. First, in Experiment 2 we used successive presentation of figures for judgment rather than simultaneous presentation. Simultaneous presentation typically leads to enhanced effects of context (Wedell \& Parducci, 1988). Thus, in Experiment 2 we explored whether contextual effects on ideals are obtained using the more typical scenario of encountering one person at a time. Second, we included items from another validated body image questionnaire so that we could see how the measure of Experiment 1 compared with these (Garner, Olmstead, \& Polivy, 1983). Finally, we included only one body type in Experiment 2.

\section{Method}

Participants and design. Participants were 83 undergraduates (27 men and 56 women), drawn from the University of Alabama, Huntsville, psychology department participant pool, who volunteered in exchange for course credit. The design consisted of three between-subjects variables: (a) range (restricted or full), (b) skew (positive or negative), and (c) judgment order (pleasantness judgments after width judgments, or vice versa). Three additional variables were manipulated within subjects: (a) target stimuli (six targets), (b) type

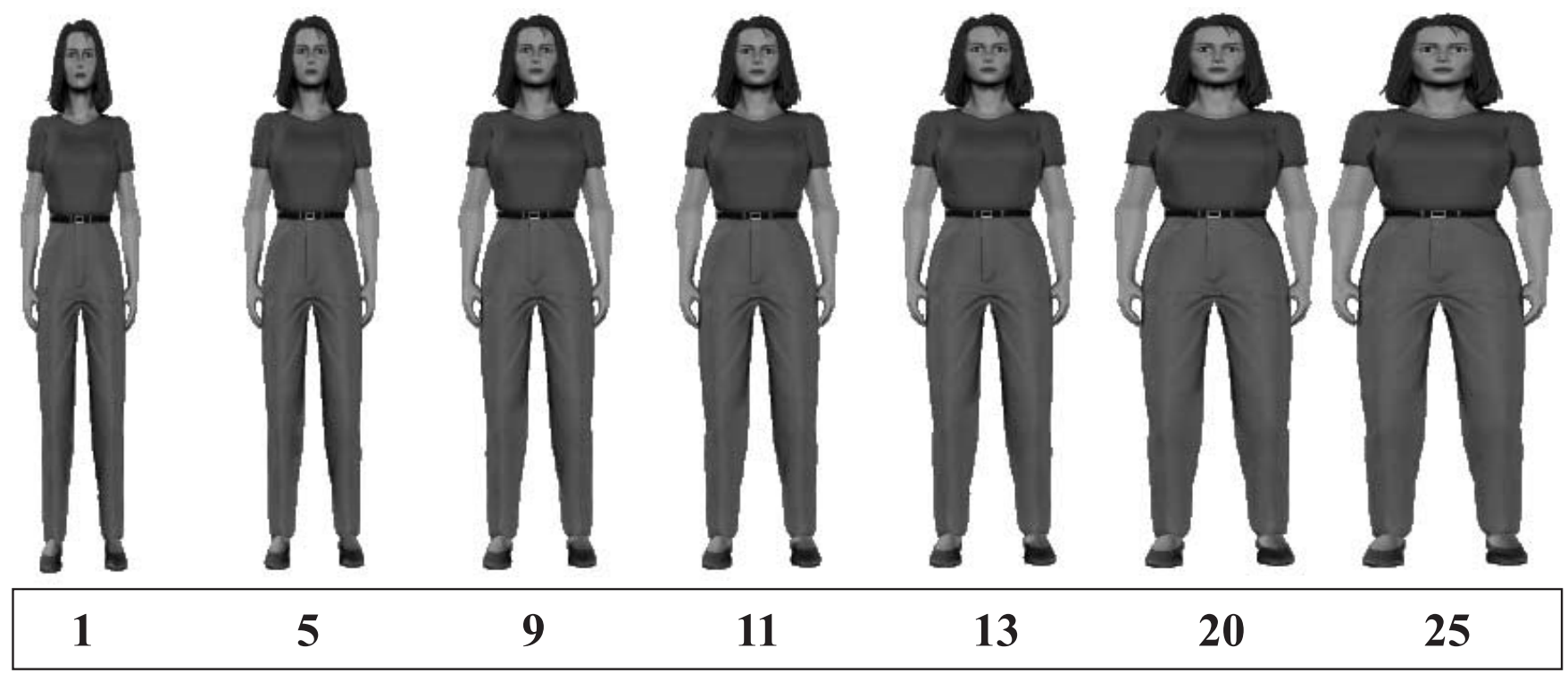

FIGURE 4 The six target body types used in Experiment 2. 
of judgment (ratings of width or pleasantness), and (c) block (four blocks of ratings for each stimulus). All variables were factorially combined.

Materials and apparatus. All instructions and stimuli were presented via desktop computers with $17-$ in. $(43-\mathrm{cm})$ color monitors, using E-prime 1.0 to display bitmap images and record judgments. Each of four identical computers was located in a single room and separated by dividers. We used three-dimensional imaging software (Poser 4.0) to create a realistic figure of a woman, dressed in green slacks and a red blouse. As in Experiment 1, size 13 represented 100\% of original size, so that the final set of 25 sizes ranged from $64 \%$ to $136 \%$ of the original width. Distributions and target sizes were the same as those used in Experiment 1.

Additional questionnaire items selected from a scale developed by Garner et al. (1983) that examined women's attitudes to their bodies were used to assess body image. Garner et al. showed that the Body Dissatisfaction subscale has high internal consistency, adequate convergent and discriminant validity, and successfully differentiates subgroups with different eating disorders from one another and from a group with normal eating habits. Statements proposed positive or negative attitudes toward body parts (e.g., stomach, legs, etc.) and asked participants to rate how often they would endorse the statement on a 6-point scale labeled as follows: $1=a l$ ways, $2=$ usually, $3=$ often, $4=$ sometimes, $5=$ seldom, and 6 $=$ never. The nine items from the Body Dissatisfaction subscale were included along with seven other items of parallel construction.

Procedure. Participants first answered questions about their age, weight, desired weight, height, and gender. After viewing instructions that briefly described the two different rating tasks, participants viewed detailed instructions about the task they would perform first (ratings of width or pleasantness). Ratings were made on the same scales used in Experiment 1 . After viewing the set of instructions for the first task, participants proceeded through the first set of 48 judgment trials (four blocks of 12 images presented in block-randomized order). The image was placed in the middle of the screen, with the rating scale printed below it. Participants simply entered the number (1-9) that corresponded to their ratings. A short delay, during which a blue screen was displayed, occurred between each trial to prevent direct visual comparison between images. After completing the first rating task, participants viewed instructions for the second task and then proceeded to complete the 48 rating trials. Participants then viewed instructions for the first set of questionnaire items (used in Experiment 1) and rated each item on the corresponding 5-point scale. Finally, participants viewed instructions for the second set of questionnaire items and rated each item on the corresponding 6-point scale.

\section{Results}

Analyses for Experiment 2 paralleled those for Experiment 1. Because all linear contrast scores calculated on participants' width judgments of the target were positive, data for all participants were retained for analysis.

Analysis of width ratings. The width ratings for the four different contextual conditions are presented in the upper panels of Figure 5. As in Experiment 1, ratings of target stimuli were higher in the positively skewed distribution than in the negatively skewed distribution, $F(1,79)=20.5, p<$ .001 , indicating contrast effects consistent with the basic predictions of range-frequency theory. Unlike Experiment 1, the Skew $\times$ Range interaction was not significant, $F(1,79)=0.6$, $p>.05$, reflecting roughly equal contrast effects in the restricted- and full-range conditions. This result indicates that in judging widths of these more realistic figures, participants were less tied to the range of values actually presented.

The range-frequency model of Equation 1 was fit to the data in the same way as in Experiment 1, with only three parameters free to vary in fitting the 48 data points. As shown in the top panels of Figure 5, the model provided an adequate fit to the data, with the proportion of variance in the mean ratings accounted for by the predicted values being .980 . The parameter values for the full-range conditions are shown in the figure. The value of $w$ was close to the values inferred for the thin and medium silhouette conditions of Experiment 1, whereas the values defining the range were somewhat more extreme and did not differ with range or skewing condition. Thus, with these more realistic and detailed figures, participants were less apt to adjust their subjective ranges to the range of values shown. Instead, these figures may have elicited the retrieval of a relevant context of body values from everyday experience. Despite the greater stability of range, ratings were highly dependent on the manipulation of frequency or rank information. Overall, the range-frequency analysis suggests that very similar processes are used to judge the widths of vague silhouettes as are used to judge the detailed realistic drawings.

Analysis of pleasantness ratings. The lower panels of Figure 5 present the pleasantness ratings for the four different contextual conditions. The rating functions exhibit the usual single-peaked form found in most attitude domains as well as the anticipated finding that the ideal body width is narrower when the context consisted of mostly narrow bodies. An ANOVA on ideals calculated as in Experiment 1 revealed a significant main effect of skew, $F(1,79)=8.0, p<$ .01 , reflecting the narrower ideal body in the positively skewed context. The Skew $\times$ Range interaction was also significant, $F(1,79)=6.5, p<.01$, reflecting the reduced contextual influence on ideals in the full range condition. As in Experiment 1, the shift in ideals shown in Figure 5 for Exper- 

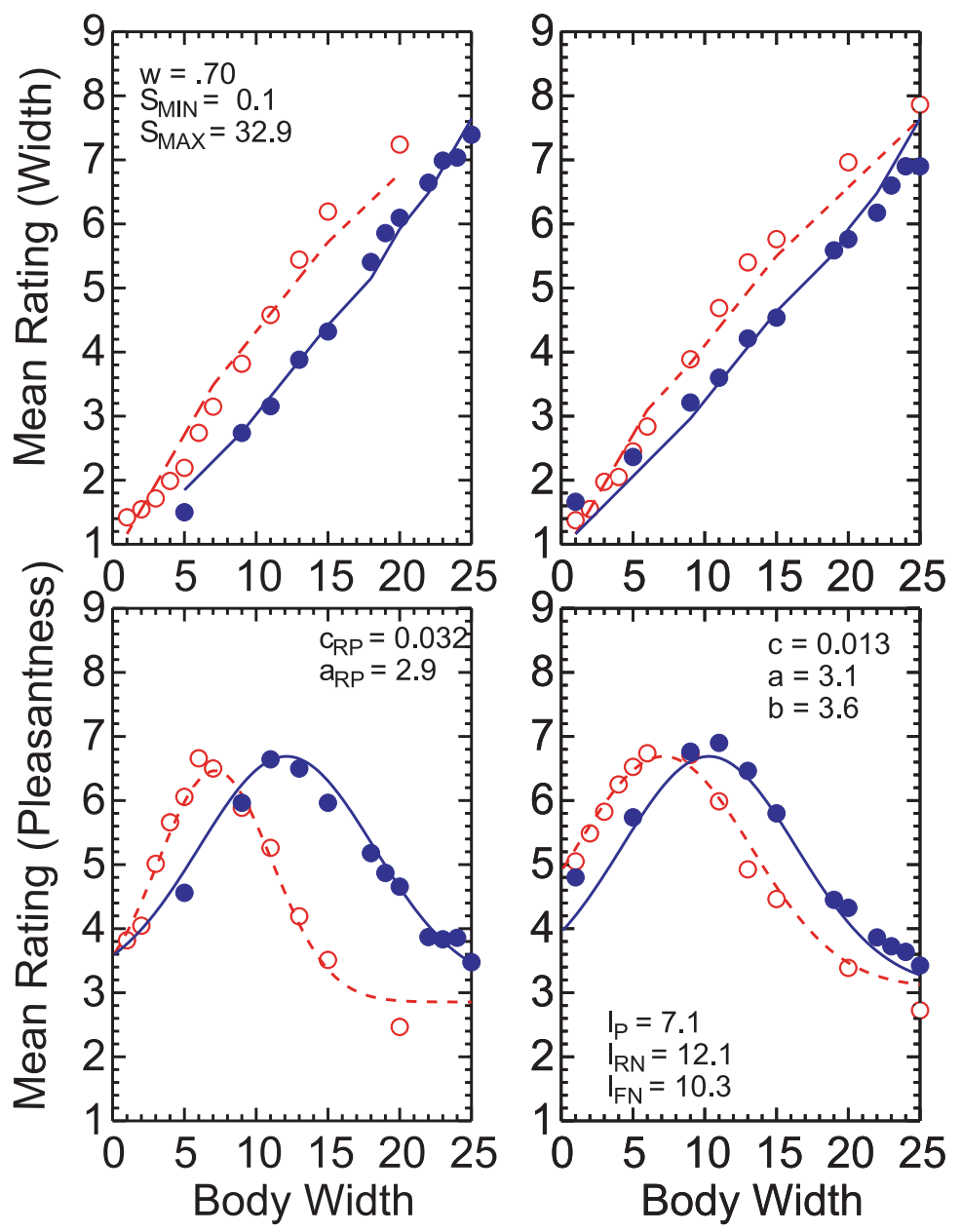

FIGURE 5 Mean ratings of width and pleasantness in the restricted-range (left panels) and full-range (right panels) condition. Data points are represented by the unfilled circles for positively skewing conditions and filled circles for negatively skewed conditions. Lines represent the fit of the theoretical models. Note $w$ is the weighting of range values, $S_{\mathrm{MIN}}$ and $S_{\mathrm{MAX}}$ are minimum and maximum values defining the subject range, respectively; $I_{P}, I_{R N}$, and $I_{F N}$ are the inferred ideals for positive skew, restricted-range negative skew and full range negative skew, respectively; $c_{R P}$ and $c$ are the values determining the narrowness of the rating functions for restricted-range positive skew and the other contextual conditions respectively; $b$ is the height of the rating function; and $a_{R P}$ and $a$ are the values for the intercepts of the rating functions, respectively.

iment 2 reflect strong reversals of preference in both restricted and full range conditions.

We again used the gaussian ideal-point model (see Equation 2) to fit the data, holding parameters constant across contexts when doing so did not significantly decrease variance explained. The final model had just eight free parameters to fit the 48 data points. Separate values of the intercept, $a$, and discriminability parameter, $c$, were required to fit the restricted range, positive-skewing condition. A single ideal was sufficient to fit data in the two positive-skewing conditions, but separate ideals were needed to fit the ideals for the two negative-skewing conditions. The eight-parameter model accounted for $96.7 \%$ of the systematic variance. The model fit indicates that the greater contextual effects on ideals for restricted-range conditions were primarily due to the greater shifts in the RN condition. An unexpected result was the greater discrimination of judgments in the RP condition (i.e., the greater value of $c$ ), indicating that the participants in this condition showed narrower latitudes of acceptability. Thus, even though ideals were inferred to be equivalent for the RP and FP conditions, the absence of extremely wide figures in the restricted range condition appeared to lead to more discriminating judgment. It is as if lack of exposure to any extreme figures on the wide end of the spectrum resulted in even small deviations from a thin ideal being deemed unacceptable.

Analyses related to the questionnaire measures. The same 8 questionnaire items were combined as in Experiment 1 to form a measure of body image. The internal consistency estimate of reliability was acceptable (Cronbach's $\alpha=$ $.88, M=0.95, S D=7.49$ ). The additional 16 body image items included in Experiment 2 were then submitted to a principal-components analysis. The final scale was based on 
TABLE 5

Principal Components Analysis (PCA) Loadings on First Component of the Body Image Instrument for the Items Retained for Analysis, Experiment 2

\begin{tabular}{cl}
\hline PCA Loadings & \multicolumn{1}{c}{ Item } \\
\hline-.79 & I think that my stomach is too big. \\
-.77 & I think that my thighs are too large. \\
.82 & I think that my stomach is just the right size. \\
-.76 & I think my hips are too big. \\
.78 & I think that my thighs are just the right size. \\
-.63 & I think my buttocks are too large. \\
.72 & I think my hips are just the right size \\
-.69 & I think my stomach is not toned. \\
.64 & I think my arms are just the right size. \\
.80 & I think that my stomach is nicely toned. \\
.74 & I like how toned my arms are. \\
.81 & I am satisfied with how toned and/or muscular my \\
\hline
\end{tabular}

the 12 items with absolute loadings of .60 or greater are shown in Table 5. Items were summed after reverse scoring, and 42 was subtracted from the total so that a score of 0 represented neutral endorsement of a positive body image, and scores could range from -30 (representing strong dissatisfaction with body image) to 30 (representing strong satisfaction with body image). These body image scores had a mean of -7.5 , a standard deviation of 15.2 , and a reliability of .93 , as estimated by coefficient alpha. As in Experiment 1, body image scores from both measures were significantly higher for men $(M=4.7$ and $M=1.5)$ than for women $(M=-0.9$ and $M$ $=-11.9), t(81)=3.4, p<.01$ and $t(81)=4.1, p<.001$.

Correlational analyses parallel to those in Experiment 1 were conducted for men and women separately and are shown in Table 6. The second body image measure, BIMAGE2, was included along with the other variables examined in Experiment 1. Unlike Experiment 1, skewing was not significantly correlated with any of the other variables. Thus, Experiment 2 did not replicate the lower body image scores associated with negative skewing found in Experiment 1; neither did it replicate the lower body image scores associated with viewing the ultrathin model as described by Stice and Shaw (1994).

The interrelations among the BIMAGE, BIMAGE2, WEIGHT, and OVERWGHT measures were as expected for both groups. The two body image measures were highly correlated for women and somewhat less so for men. Participants with higher body image scores reported weighing less and reported themselves less overweight. This was true for both men and women, although the strength of these relationships was greater for women. It is interesting to note that in both Experiment 1 and 2 the correlation between perceptions of being overweight and one's reported weight was extremely high for women (close to .90) and more moderate for men (closer to .60). This extremely high correlation for women indicates that, regardless of their own body weights,
TABLE 6

Correlations Among Key Participant and Judgment Variables for Women and Men, Experiment 2

\begin{tabular}{lcccccc}
\hline Variable & 1 & 2 & 3 & 4 & 5 & 6 \\
\hline Women $(N=56)$ & & & & & & \\
1. BIMAGE & - & & & & & \\
2. BIMAGE2 & $.85^{* * * *}$ & - & & & & \\
3. OVERWGHT & $-.52^{* * * *}$ & $-.56^{* * * *}$ & - & & & \\
4. WEIGHT & $-.45^{* * * *}$ & $-.50^{* * * *}$ & $.91 * * * *$ & - & & \\
5. IDEALDEV & $.28^{* *}$ & $.26^{*}$ & -.11 & -.17 & - & \\
6. Skew & -.05 & -.04 & .01 & .08 & -.08 & - \\
Men $(N=27)$ & & & & & & \\
1. BIMAGE & - & & & & & \\
2. BIMAGE 2 & $.66^{* * * *}$ & - & & & & \\
3. OVERWGHT & $-.68^{* * * *}$ & $-.66^{* * * *}$ & - & & & \\
4. WEIGHT & $-.73^{* * * *}$ & $-.47^{* *}$ & $.61 * * * *$ & - & & \\
5. IDEALDEV & -.10 & .06 & .26 & -.04 & - & \\
6. Skew & -.01 & .02 & .08 & -.16 & .13 & - \\
\hline
\end{tabular}

Note. $\quad$ BIMAGE = body image scale from Experiment 1; BIMAGE2 = second body image scale developed for experiment 2 ; OVERWGHT $=$ perception of being overweight (actual weight minus ideal weight); WEIGHT = participant's actual weight; IDEALDEV = deviation score of ideal weight inferred from participant's judgments and average ideal in the corresponding contextual condition.

$* p<.06 . * * p<.05 . * * * p<.001$.

women tended to project the same slim ideal weight for themselves $(M=125, S D=14.7)$, whereas men showed more variability in ideal body weights projected for themselves $(M$ $=170, S D=21.4)$. This relationship was most apparent at the extreme end of the weight continuum. The three women who reported weighing $200 \mathrm{lbs}$ or more indicated a mean ideal weight of only $133 \mathrm{lbs}$, whereas the four men who reported weighing $200 \mathrm{lbs}$ or more indicated a mean ideal weight of $201 \mathrm{lbs}$. The significant positive correlation between body image scores and the IDEALDEV measure for women found in Experiment 1 was replicated, indicating that those who scored low in body satisfaction tended to use very thin ideal ideals in evaluating the attractiveness of the body images.

Generality of judgment results across groups. We again examined whether contextual effects on judgments of the experimental stimuli were similar across groups. To facilitate comparison with Experiment 1, we grouped women on the first body image measure. Our three subgroups were (a) women with low body image scores (BIMAGE $<-1.0, n=$ 27), (b) women with high body image scores (BIMAGE $^{3}$ $-1.0, n=29)$, and (c) men $(n=27)$. We conducted ANOVAs on the mean width judgments of the target stimuli and on the inferred ideals for each participant. In the domain of width judgment, each group showed a significant main effect of skewing $(p<.05)$, consistent with results reported earlier for the group as a whole. In the domain of pleasantness judgments, once again the women with low body image scores showed no significant effects of skewing on inferred ideals, but those with high body image scores did. Although men did 
not show a main effect of skewing on body image, they did show a Skewing $\times$ Range interaction. Only in the restricted range condition did they show a strong effect of context on ideal-point locations, consistent with the group data. Thus, as in Experiment 1, body satisfaction moderated the effects of context on ideals.

\section{Discussion}

Experiment 2 generalized the results of Experiment 1 in several ways. First, the same strong effects of context on width and pleasantness judgments were observed in both experiments. Thus, these effects do not depend on the use of silhouettes or simultaneous presentation but generalize to the use of more realistic drawings presented for successive judgment. Second, in both experiments women who viewed their own body images as inadequate tended to use significantly thinner ideals in judging other body images. Third, analyses of judgment data by group in both experiments showed that women with lower body satisfaction scores did not shift the ideals they used to judge body images with context, but women with higher body image scores and men did. Thus, body satisfaction moderated the contextual effects on ideals.

One aspect of the data that did not generalize was the relationship between the contextual manipulation and reported body satisfaction for women. Prior research had indicated that women exposed to a glamorous, ultrathin model rated their own body images lower than women exposed to a model who was more moderate in weight and less glamorous (Stice \& Shaw, 1994; Thornton \& Maurice, 1999). Experiment 1 demonstrated the opposite effect of exposure to thin images, with women in the positively skewed condition rating themselves higher on the body image measure than those in the negatively skewed condition. Experiment 2 showed no linkage between the skewing manipulation and reported body image. These inconsistent results indicate that there is no clear linkage between experimental manipulation of body images individuals view and their self-reported body satisfaction. As noted earlier, Henderson-King and Henderson-King (1997) found individual differences in how exposure to thin figures affected ratings of body dissatisfaction. These results underscore the lack of consistent effects.

\section{GENERAL DISCUSSION}

A key contribution of these experiments is that they provide the strongest evidence to date that mere exposure to a skewed set of extremely thin human figures leads to the adoption of a thin ideal for evaluating body images. This effect is demonstrated quite clearly in the shifts in the pleasantness ratings of body images shown in Figures 2, 3, and 5. Although mechanisms such as reinforcement of a thin body ideal (through associated positive attributes) may play an important role in shifting preference toward a thin ideal in real world applications, the results of Experiments 1 and 2 show that reinforcement is not necessary to achieve such a shift. The fact that the patterns of context effects on width judgments differed from those on pleasantness judgments supports the idea that the mechanism guiding changes in ideals is not strictly mediated by implicit width judgments but may involve separate retrieval processes for determining category prototypes.

Previous research has shown that exposure to pictures of thin models can result in lowered body satisfaction (Stice \& Shaw, 1994; Thornton \& Maurice, 1997), although Henderson-King and Henderson-King (1997) found that this occurred only for heavier women, with the opposite effect observed for lighter women. In contrast to the typical finding, Experiment 1 found that exposure to thinner silhouettes led to increased body satisfaction for women, and Experiment 2 found no effect of exposure to the thinner set of figures on either of two body satisfaction questionnaires. Thus, the relationship between body satisfaction rating and exposure to thin body images is not always consistent. One possibility is that the typical finding is not guided by the use of a thinner ideal but rather is a result of a contrast effect, as Thornton and Maurice $(1997,1999)$ have posited, so that women feel less adequate when they compare themselves to women whom they perceive as more glamorous or beautiful.

Two consistent findings across Experiments 1 and 2 are also particularly noteworthy. First, in both experiments the body satisfaction measures correlated positively with the inferred ideals women used in judging body images (the IDEALDEV measure). This meant that women who were more dissatisfied with their own bodies consistently used thinner ideals in judging body images. This result lends support to the idea that women with negative body images may maintain those negative self-appraisals by holding onto an unrealistically thin ideal used to judge others and themselves. A second important finding is that women who were more dissatisfied with their own bodies were insensitive to the skewing manipulation when judging the pleasantness of other body images. This was not because they were insensitive to the contextual manipulation on the whole, as they did show significant effects of skewing on width judgments. Instead, this finding supports the idea that dissatisfaction with one's own body image may be due to a persistent tendency to recruit an ultrathin body ideal regardless of recent contextual experiences. This finding is troubling, because it implies that merely exposing women who are dissatisfied with their own bodies to wider body types may do little to move them away from a thin ideal. Instead, they may ignore cues from their environment that are inconsistent with the thin ideal and continue to maintain harsh judgments of normal body images.

Our results also indicate a possible new and direct measure of endorsement of a thin ideal that could be used in future research in this area. Rather than simply having women evaluate their dissatisfaction with their own body image, 
women could rate the attractiveness of different body images, such as silhouettes that vary only in width. This procedure allows for the more precise measurement of the ideals they use in evaluating other body images. Furthermore, the distribution of images could be varied within subjects to examine how sensitive their ideals are to contextual manipulation. Women who adopt an ultrathin ideal that is insensitive to contextual manipulation might be more at risk of eating disorders and other negative outcomes.

Finally, we note that our study was consistent with other studies in the body image literature that reveal generally greater dissatisfaction of women with their body image. In our sample, women were significantly more dissatisfied with their bodies than were men, and women felt themselves to be significantly more overweight. The dissatisfaction of women with their body image has been reported by many researchers (Adams, Katz, Beauchamp, Cohen, \& Zavis, 1993) and is a cause for great concern. Several researchers have now shown that the adoption of the thin ideal is a plausible mediator of eating disorder symptomatology (Barber, 1998; Botta, 1999; Harrison, 1997; and Stice, 1994). McCarthy (1990) speculated that the cultural ideal of thinness for women may be the cause of greater rates of depression in women than in men. Our research did not attempt to examine these linkages, but rather demonstrates a clear linkage between exposure to thin images and the adoption of a thinner ideal used to evaluate body images. Although other associative mechanisms are undoubtedly involved in perpetuating the thin ideal in our culture, the current findings boost the claim that mass media presentation of positively skewed distributions of body images is one way this cultural norm is perpetuated. These findings also suggest that inclusion of a fuller range of body images in the media may reduce these effects of skewing and lead to an ideal that is closer to the norm, at least for persons who are not already strongly dissatisfied with their body images.

\section{ACKNOWLEDGMENTS}

This research was supported by NSF grant \#SBR-9911132. The authors would also like to thank Matt Cornwall and Diana Riser for their assistance with Experiment 2.

\section{REFERENCES}

Adams, P. J., Katz, R. C., Beauchamp, K., Cohen, E., \& Zavis, D. (1993). Body dissatisfaction, eating disorders, and depression: A developmental perspective. Journal of Child \& Family Studies, 2(1), 27-46.

Barber, N. (1998). The slender ideal and eating disorders: An interdisciplinary "telescope" model. International Journal of Eating Disorders, 23, 295-307.

Birnbaum, M. H. (1974). Using contextual effects to derive psychophysical scales. Perception \& Psychophysics, 15, 89-96.
Botta, R. A. (1999). Television images and adolescent girls' body image disturbance. Journal of Communication, 49, 22-41.

Brown, T. A., Cash, T. F., \& Mikulka, P. J. (1990). Attitudinal body-image assessment: Factor analysis of the Body-Self Relations Questionnaire, Journal of Personality Assessment, 55, 135-144.

Cash, T. F. (1994). The Situational Inventory of Body Image Dsyphoria: Contextual assessment of a negative body image. The Behavior Therapist, 17, $133-134$.

Fouts, G., \& Burggraf, K. (1999). Television situation comedies: Female body images and verbal reinforcements. Sex Roles, 40, 473-481.

Garner, D. M., Olmstead, M. P., \& Polivy, J. (1983). Development and validation of a multidimensional eating disorder inventory for anorexia nervosa and bulimia. International Journal of Eating Disorders, 2, 15-34.

Harrison, K. (1997). Does interpersonal attraction to thin media personalities promote eating disorders? Journal of Broadcasting \& Electronic Media, 41, 478-500.

Henderson-King, E., \& Henderson-King, D. (1997). Media effects on women's body esteem: Social and individual difference factors. Journal of Applied Social Psychology, 27, 399-417.

Kahneman, D., \& Miller, D. T. (1986). Norm theory: Comparing reality to its alternatives. Psychological Review, 93, 136-153.

Kalodner, C. R. (1997). Media influences on male and female non-eating-disordered college students: A significant issue. Eating Disorders: The Journal of Treatment and Prevention, 5, 47-57.

Kilbourne, J. (1994) Still killing us softly: Advertising and the obsession with thinness. In P. Fallan, M. A. Katzman, \& S. C. Wooley (Eds.), Feminist perspectives on eating disorders (pp. 395-418). New York: Guilford.

Lambert, A. J., \& Wedell, D. H. (1991). The self and social judgment: Effects of affective reaction and "own position" on judgments of ambiguous and unambiguous information about others. Journal of Personality and Social Psychology, 61, 884-897.

McCarthy, M. (1990). The thin ideal, depression and eating disorders in women. Behaviour Research and Therapy, 28, 205-215.

Mellers, B. A., \& Birnbaum, M. H. (1983). Contextual effects in social judgment. Journal of Experimental Social Psychology, 19, 157-171.

Murray, S. H., Touyz, S. W., \& Beumont, P. J. V. (1996). Awareness and perceived influence of body ideals in the media: A comparison of eating disorder patients and the general community. Eating Disorders: The Journal of Treatment and Prevention, 4, 33-46.

Parducci, A. (1965). Category judgments: A range-frequency model. Psychological Review, 72, 407-418.

Parducci, A. (1995). Happiness, pleasure and judgment: The contextual theory and its applications. Mahwah, NJ: Lawrence Erlbaum Associates, Inc.

Parducci, A., \& Wedell, D. H. (1986). The category effect with rating scales: Number of categories, number of stimuli, and method of presentation. Journal of Experimental Psychology: Human Perception and Performance, 12, 496-516.

Riskey, D. R., Parducci, A., \& Beauchamp, G. K. (1979). Effects of context in judgments of sweetness and pleasantness. Perception \& Psychophysics, 26, 171-176.

Smith, R. H., Diener, E., \& Wedell, D. H. (1989). Intrapersonal and social comparison determinants of happiness: A range-frequency analysis. Journal of Personality and Social Psychology, 56, 317-325.

Spitzer, B. L., Henderson, K. A., \& Zivian, M. T. (1999). Gender differences in population versus media body sizes: A comparison over four decades. Sex Roles, 40, 545-565.

Stice, E. (1994). Review of the evidence for a sociocultural model of bulimia nervosa and an exploration of mechanisms of action. Clinical Psychology Review, 14, 633-661.

Stice, E. (1998). Modeling of eating pathology and social reinforcement of the thin-ideal predict onset of bulimic symptoms. Behavior Research and Therapy, 36, 931-944.

Stice, E., Schupak-Neuberg, E., Shaw, H. E., \& Stein, R. I. (1994). Relation of media exposure to eating disorder symptomatology: An exami- 
nation of mediating mechanisms. Journal of Abnormal Psychology, 103, 836-840.

Stice, E., \& Shaw, H. E. (1994). Adverse effects of the media portrayed thin ideal on women and linkages to bulimia symptomatology. Journal of Clinical and Social Psychology, 13, 288-308.

Thornton, B., \& Maurice, J. K. (1997). Physique contrast effect: Adverse impact of idealized body images for women. Sex Roles, 37, 433-439.

Thornton, B., \& Maurice, J. K. (1999). Physical attractiveness contrast effect and the moderating influence of self-consciousness. Sex Roles, 40, 379-392.

Wedell, D. H. (1994). Contextual contrast in evaluative judgments: Test of pre- versus post-integration models of contrast. Journal of Personality and Social Psychology, 66, 1007-1019.
Wedell, D. H. (1996). A constructive-associative model of the contextual dependence of unidimensional similarity. Journal of Experimental Psychology: Human Perception and Performance, 22, 634-661.

Wedell, D. H., \& Parducci, A. (1988). The category effect in social judgment: Experimental ratings of happiness. Journal of Personality and Social Psychology, 55, 341-356.

Wedell, D. H., \& Pettibone, J. C. (1999). Preference and the contextual basis of ideals in judgment and choice. Journal of Experimental Psychology, 128, 346-361.

Wedell, D. H., Parducci, A., \& Geiselman, R. E. (1987). A formal analysis of ratings of physical attractiveness: Successive contrast and simultaneous assimilation. Journal of Experimental Social Psychology, 23, 230-249. 